https://doi.org/10.31713/m913

\title{
DEVELOPMENT OF INTELLIGENT SYSTEMS FOR OPTIMAL PROCESS CONTROL
}

\author{
Suleimenov B. A. \\ Satbayev University, Doctor of Engineering Sciences, Professor, \\ Head of Department "Automation and Control”, Kazakhstan
}

Kulakova E.A.

Satbayev University, Master of Engineering and Technology, Doctoral Student of Department "Automation and Control”, Kazakhstan

\begin{abstract}
The search for resource-saving technologies implies not only research in the field of new methods of processing raw materials, but also new approaches to managing technological processes. Another important way of resource saving is the ability to quickly assess the technical condition of equipment, which will allow to timely prevent the emergency state of the main and auxiliary technological equipment. The paper deals with the development of intelligent control systems and diagnostics of processes using fuzzy logic, neural networks and hybrid models for creating control algorithms. The subject of the research is control algorithms developed to determine the key variables of the copper melting process in a liquid bath and algorithms for evaluating the technical condition of turbine units. All algorithms are developed on the basis of PFE matrices compiled by experienced technology experts during a "thought" experiment. The resulting models are carefully studied and analyzed for their sensitivity, stability, and single-valuedness. The absolute error of discrepancy between experimental and calculated variables became the criterion of adequacy.

Finally, the conducted researches have shown high efficiency of the control algorithms, obtained by using the artificial intelligence methods. In comparison with classical methods of building analytical and statistical models, methods based on the knowledge and experience of human experts allow creating optimal control systems for complex technological processes significantly easier, faster and more efficient.
\end{abstract}

\section{Introduction}

One of the main ways of resource saving in the mining and metallurgical industries is the introduction of automated systems of optimal process control (APCS) at industrial enterprises in these industries, which allow more efficient process control with the least loss of material, heat, electricity, labour and other resources of an enterprise. 
The development of optimal control systems began in the 60 s of the last century. However, so far in the world, few effective automated control systems have been implemented - the main reason is that it is not possible to create adequate mathematical models of technological processes due to the extreme complexity of modern technologies.

The rapid surge in the theory and practice of artificial intelligence (AI) methods in recent years has allowed us to find a simpler and more effective way to develop APCS using modern intelligent technologies. Let's consider the use of AI methods for the synthesis of automated control systems.

\section{The concept of creating intelligent control systems}

The analysis of works in the field of theory and practice of artificial intelligence has shown that currently there have been developed effective artificial intelligence technologies that are used in various practical applications, including control. However, most authors use these technologies for the development, research, and implementation of local control systems designed primarily to solve problems of stabilizing certain process variables.

We have not been able to find in the literature any examples of using intelligent technologies (IT) for creating optimal process control systems. As noted above, traditional methods of developing sufficiently adequate mathematical models of processes have not allowed the implementation of any significant control system in practice (at least in Kazakhstan).

In this regard, in this work we set the goal to further improve and develop information technologies, with the account of the specific features of technological process control.

Numerous studies conducted by our department, as well as the analysis of publications, have shown that IT can be used in the development of a straight optimal process control model, rather than a model of the technological process itself. That is the considered technologies allow immediate developing control algorithms, in contrast to the traditional chain: development of a process model structure $\rightarrow$ experimental research on site $\rightarrow$ model identification $\rightarrow$ formulation of optimization problem $\rightarrow$ selection of optimization method $\rightarrow$ development of an optimal control algorithm. The traditional approach assumes a long (sometimes taking several years), expensive and not always successful way of development of an optimal control system. 
Employing IT allows solving similar tasks immediately, and as experience has shown, quite successfully. The fact is that the methods of artificial intelligence involve using the knowledge, experience and intuition of human experts who are familiar with the subject area. In other words, the so-called "ready knowledge" effect is used here. In contrast, the development of a mathematical model (the main component of the system) is a process of creating "new knowledge", and therefore requires quite a long time for conducting theoretical research, as well as large material and labour costs for conducting empirical research and identifying the model.

Using the main idea of the work (development of a process control model instead of a process model) and developing existing IT methods, we propose the following three-stage procedure for creating optimal process control systems.

At the first stage, a priori studies of the technological features of the control object are carried out; they are based on literary sources, publications in periodicals and factory technical documentation. As a rule, existing technological processes had to go through a long stage of scientific research, pilot and industrial tests before they were implemented into production. There are probably still materials from these studies, as well as attempts to create mathematical models of this process. A thorough analysis of all this information is necessary in order to use it in the development of intelligent control systems.

At the same stage, it is necessary to analyze the process under study as a control object with the identification of input and output, controlled and uncontrolled, managed and unmanaged variables. It is necessary to estimate the response rate of an object, and an object's type (continuous or discrete), the degree of information entirety of variables, the operating range of the variable change of an object, etc.

After a thorough analysis of the available information, it is necessary to draw up the structure of the future control system, which will greatly facilitate further work.

At the second stage, a model of the control process is developed. With the help of experienced experts (process operators, or engineers and technicians of a workshop or factory), the main control goal (analogous to the target function in optimization problems) is determined; it is usually known, and experienced operators usually strive to achieve it. Then, using the ranking method, the main (according to 
experts) variables for this object (process) from the general list of all types of variables are determined.

The main task of the second stage is to compile a matrix for planning a complete factorial (CF). The CF matrix and a three-level evaluation of input and output variables are used to create an object (process) control model.

In this case, for three-level factors, the total number of possible combinations of the number of factors for two input variables is $N=3^{2}=9$, for three variables $-3^{3}=27$, and so on.

For example, with two input variables, a PFE planning matrix is compiled, shown in table 1 . Tables of this type are the basis for the development of intelligent systems, as they focus many years of experience, knowledge and intuition of people who are experts in a particular subject area.

Table 1

PFE planning Matrix

\begin{tabular}{l|l|l|l}
\hline $\begin{array}{l}\text { The number of the exper- } \\
\text { iment }\end{array}$ & $X_{1}$ & $X_{2}$ & $Y^{\curvearrowright}$ expert assessment \\
\hline 1 & 0,0 & 0,0 & \\
\hline 2 & 0,0 & 0,5 & \\
\hline 3 & 0,0 & 1,0 & \\
\hline 4 & 0.5 & 0,0 & \\
\hline 5 & 0.5 & 0,5 & \\
\hline 6 & 0.5 & 1,0 & \\
\hline 7 & 1,0 & 0,0 & \\
\hline 8 & 1,0 & 0,5 & \\
\hline 9 & 1,0 & 1,0 & \\
\hline
\end{tabular}

Values: $0.0 ; 0.5 ; 1.0$ mean the minimum, average, and maximum values of the input variables $X_{1}$ and $X_{2}$. The expert can only use his experience, knowledge and intuition to set the values of the output variable $Y^{E}$ (control action) in the range from 0,0 to 1,0 . Normalization in the range from 0 to 1 of the input and output variables is performed by the formula

$$
\bar{x}=\frac{x-x_{\min }}{x_{\max }-x_{\min }},
$$

где $\bar{x}$ - normalized value of the input or output variable; $x$ - running value of the variable; $x_{\min }, x_{\max }-$ minimum and maximum value of the variable. 
Compiling an experiment planning matrix is much more convenient for experts than drawing up rules for fuzzy products of the type recommended in all textbooks and publications. At the same time an expert does not need to invent infinite terms: "very much", "very, very little", "quite sufficient", etc. He simply puts the value of the output (control) variable in the range from 0.0 to 1.0. And the CF planning matrix can be used for three different methods of creating a control model: expert systems, neural networks, and neuro-fuzzy algorithms.

In contrast to the well-known classical method of experiment planning, drawing up the $\mathrm{CF}$ planning matrix with the help of experts significantly speeds up and reduces the cost of this procedure. Experts conduct so-called "mental experiments" instead of expensive, actually conducted active experiments. In addition, it should be taken into account that conducting active experiments under the live production conditions is unrealistic due to the possible emergency situations when changing process variables from their minimum values to their maximum values, and vice versa. In addition, many enterprises simply do not have possibilities to change variables according to the $\mathrm{CF}$ planning matrix.

It should be emphasized that $Y_{i}$ output values are actually controlling variables, so the planning matrix displays the process control model for all the combinations of input variables planned by experts. To calculate values in intermediate combinations of input variables, it is necessary to synthesize a process control model, which is the main task of the second stage.

It should be noted that it is most effective to use well-known mathematical dependencies identified at the first stage of research along with intelligent models. At the same time, it is necessary to make sure that such dependencies adequately reflect certain physical and chemical laws of a particular process.

At the third stage, the created control models are studied. At that the following actions are performed.

The resulting models are carefully studied and analyzed for their sensitivity, stability, and single-valuedness. For this purpose, the control process is modelled for various changes in input variables; curves are drawn for changes in output variables on the account of input variables changes, and their analysis is performed employing experts. 
After the study of models obtained by different methods is completed, a comparative analysis for their adequacy is carried out. For this purpose, models are used to calculate the output variables according to the values of input variables taken from the $\mathrm{CF}$ planning matrix and compare them with the estimates provided by the expert. After that, a comparison matrix is formed (see Table 2), which allows calculating the value of the modelling error by various means.

The absolute error defined as

$$
\delta=100 \frac{1}{N-1} \sum_{i=1}^{N}\left|Y^{E}-Y^{C}\right|,
$$

где $Y^{E}$ и $Y^{C}$ - experimental and calculated values of output variables respectively.

Table 2

Matrix of comparison of calculated and experimental values of the output value

\begin{tabular}{c|l|l|l|l}
\hline The number of the experiment & $X_{1}$ & $X_{2}$ & $\begin{array}{l}Y^{C} \text { model } \\
\text { value }\end{array}$ & \begin{tabular}{l}
$Y^{E}$ value expert \\
\hline 1
\end{tabular} \\
\hline 2 & 0,0 & 0,0 & & \\
\hline$\cdots$ & 0,0 & 0,5 & & \\
\hline 9 & 0,0 & 1,0 & & \\
\hline
\end{tabular}

The absolute error is calculated for models obtained using three different approaches, and then their comparative analysis is performed. The model with the smallest absolute error is considered the most appropriate.

The most appropriate model is to undergo simulation tests under the live production conditions. At that, the model input is supplied with real input variables taken from the measuring equipment of the industrial unit, and the simulation results (the output control variable) are compared to the control value actually carried out by an experienced operator-technologist. If the simulation test results are satisfactory, the model is integrated into the industrial controller. Otherwise, the process starts from the beginning - returning to the first stage, and updating all of the model parameters.

Further, the paper shows how the proposed concept is used for the synthesis of an optimal control system for the process of copper melting in a liquid bath. 


\section{Development of an optimal control system for the process of copper melting in a liquid bath}

When considering the technology for processing copper concentrates in a liquid bath furnace (LBF), it is necessary to take into account the fact that this process is relatively new, has not yet been sufficiently studied and has been implemented only at a few facilities of non-ferrous metallurgy enterprises in the world.

\subsection{Characteristics of the LBF process as an object of control}

The object of control is a LBF industrial unit. The LBF industrial unit is a two-zone caisson furnace with continuous roof charging into the melting zone, side blast into the melt and continuous output of melting products from the reaction zone.

The charge is prepared in advance for the entire campaign based on calculations of the melting mode and data on the composition of processed materials. Once in the melt, the charge is heated and dissolved, the higher sulphides of the charge dissociate and form the simplest sulfide compounds and elemental sulfur. The products of dissociation interact with the oxygen of the blast bubbling the melt, resulting in the formation of matte, slag and sulfurous anhydride rich gases. The matte-slag emulsion gradually delaminates as it descends down. The matte forms a continuous given phase, and the slag enters the reduction zone through the siphon, after which it flows through the second siphon into the electric slag settling tank. Periodically, as the matte accumulates, it is released from the oven into a bucket and poured into the molds, after being weighed.

The slag from the electric settling tank is also released into a bucket and poured into the molds. After solidification, the melting products are taken out of the shop.

The recovery zone is similar in design to the melting zone and is used for studying the modes of processing ores containing zinc and lead; in other cases it is used as a flow container.

The furnace gases are removed from each zone through flue ducts and sent to a system consisting of a cyclone filter, scrubber, $\mathrm{CO}$ afterburning and cooling chambers, and enter either the chimney or the system for obtaining elemental sulfur.

From the characteristics of the process of processing materials in the liquid bath furnace at Balkhash Mining-and-Metallurgical Integrated Works, it can be concluded that the control object is a techno- 
logical process with a complex relationship of its characteristic parameters. Among the features of the LBF at Balkhash Mining-andMetallurgical Integrated Works the following should be highlighted:

- a small response rate of the object along the channel: blast - the temperature of the melt and waste gases; and a relatively large one along the channel: blast, the composition of the input products - the composition of matte and slag;

- the object can be classified as continuous (continuous feed of charge, blast, continuity of basic transformations (heating, melting, oxidation, recovery of materials, mass and heat exchange) continuous release of slag and periodic release of matte, continuous exhaust of waste gases);

- multidimensionality of the technological process characterized by a large number of input and output variables;

- non-stationary process parameters due to fluctuations in the values of input variables because of insufficient charge averaging;

- incomplete information (the presence of periodically controlled parameters, as well as delays both on the object itself and in the measurement channels, a significant level of interference in the latter, etc.);

- narrow operating range of the melt temperature in the melting zone;

- the processes of processing various types of raw materials studied at the LBF unit have significant differences in the nature of the physical and chemical phenomena.

\subsection{Development of models for control the LBF process based on the PFE matrix}

The using of intelligent technologies allows us to solve problems of synthesis of optimal control algorithms immediately, and as experience has shown, quite successfully. With the help of experienced experts (operators-technologists, or engineers of a workshop or factory), it is necessary to determine the main goal of management (analogous to the target function in optimization problems), which is usually known and which experienced operators usually strive to achieve. Thereafter, using the ranking method, from the general list of all types of variables, those that, according to experts, are the main ones for this object (process) are determined. 
The main task in the development of the control model is creation of matrix for planning a complete factor experiment (PFE). The quality of the PFE matrix significantly affects the efficiency of the entire control system. The PFE planning matrix should reflect the experience, knowledge, and intuition of process operators. The performance of the entire control system will depend on the quality of the PFE matrix.

The task of the automated process control system is determination of the optimal process management modes: Y1, Y2 and Y3, depending on the composition of the initial charge: $\mathrm{X} 1, \mathrm{X} 2, \mathrm{X} 3$ and $\mathrm{X} 4$. As a rule, such calculations must be made once per shift. The survey of the shop's technologists allowed to create a matrix of PFE planning for 81 experiments with a three-level assessment $(0,0 ; 0,5$ and 1,0$)$, four input variables: $N=3^{4}=81$. For more accurate modeling of the control process, two additional levels were added: 0.25 and 0.75 . Since the number of experiments would increase many times, these levels were entered only for the normal conduct of the process. Consequently, combinations were obtained for 89 experiments (table 3).

Table 3

Fragment of the PFE planning matrix

\begin{tabular}{l|l|l|l|l|l|l|l}
\hline \multirow{2}{*}{ № } & \multicolumn{6}{|l|}{ Input variable } & \multicolumn{2}{l}{ Output variable } \\
\cline { 2 - 8 } & $\begin{array}{l}\text { copper } \\
\text { content } \\
\text { in the } \\
\text { charge } \\
X_{1}\end{array}$ & $\begin{array}{l}\text { sulfur } \\
\text { content in } \\
\text { the } \\
\text { charge } X_{2}\end{array}$ & $\begin{array}{l}\text { silicon } \\
\text { oxide } \\
\text { content in } \\
\text { the } \\
\text { charge } X_{3}\end{array}$ & $\begin{array}{l}\text { magnetite } \\
\text { content in } \\
\text { the } \\
\text { charge } X_{4}\end{array}$ & $\begin{array}{l}\text { lonsumpt } \\
\text { ion of } \\
\text { blast } Y_{1}\end{array}$ & $\begin{array}{l}\text { the flow } \\
\text { rate of } \\
\text { concen- } \\
\text { trate } Y_{2}\end{array}$ & $\begin{array}{l}\text { oxygen } \\
\text { enrich- } \\
\text { ment of } \\
\text { the blast } \\
Y_{3}\end{array}$ \\
\hline 1 & 0 & 0 & 0 & 0 & 0,12 & 0,87 & 0,18 \\
\hline 2 & 0 & 0 & 0,5 & 0 & 0,37 & 0,62 & 0,25 \\
\hline 3 & 0 & 0,5 & 0 & 0 & 0,37 & 0,62 & 0,31 \\
\hline 4 & 0 & 0,5 & 0,5 & 0 & 0,5 & 0,62 & 0,62 \\
\hline$\ldots$ & $\ldots$ & $\ldots$ & $\ldots$ & $\ldots$ & $\ldots$ & $\ldots$ & $\ldots$ \\
\hline 86 & 1 & 1 & 0 & 1 & 0,43 & 0,43 & 0,62 \\
\hline 87 & 1 & 1 & 1 & 1 & 1 & 0,68 & 0,81 \\
\hline 88 & 1 & 1 & 0,5 & 1 & 0,75 & 0,56 & 0,75 \\
\hline 89 & 1 & 0,5 & 1 & 1 & 0,87 & 0,62 & 0,62 \\
\hline
\end{tabular}

It should be emphasized that in table 3, all variables had been normalized in the range from 0.0 to 1.0 . The variables had been recalculated in accordance with formula 1.35, while the maximum and minimum values of input and output variables were determined from practice. 
The PFE planning matrix is compiled by experienced technologists using a "thought" experiment. Therefore, compiling such a matrix is significantly simpler than according to an active experiment. Table 3 focuses on the long-term experience of technologists at the melting furnace in Balkhash.

The PFE planning matrix can be used in the development of control models in three ways: experiment planning method, fuzzy modeling method, neural network method, and neuro-fuzzy methods. The creation of models for controlling the melting of copper in PV using each of these methods is discussed below.

\subsection{Synthesis of a fuzzy control model}

The fuzzy model was developed using Matlab graphical tools. Then the membership functions for three input and two output variables were defined. For this purpose, the Matlab membership function editor was used. Then the fuzzy production rules were formed. At the same time, each experiment from the PFE planning matrix corresponded to its own rule. For example, for experiments №1, №2 и №3 , the production rule looks like this:

RULE 1: "IF COPPER CONTENT IN THE CHARGE IS THE MINIMUM" AND "SULPHUR CONTENT IN THE CHARGE IS THE MINIMUM" AND "SILICON OXIDE CONTENT IN THE CHARGE IS THE MINIMUM" AND "MAGNETITE CONTENT IN THE CHARGE IS THE MINIMUM" THEN "THE CONSUMPTION OF BLAST IS 0.12" AND "THE FLOW RATE OF CONCENTRATE IS 0.87" AND "OXYGEN ENRICHMENT OF THE BLAST IS 0.18".

RULE 2: "IF COPPER CONTENT IN THE CHARGE IS THE MINIMUM" AND "SULPHUR CONTENT IN THE CHARGE IS THE MINIMUM" AND "SILICON OXIDE CONTENT IN THE CHARGE IS THE MEDIUM" AND "MAGNETITE CONTENT IN THE CHARGE IS THE MINIMUM" THEN "THE CONSUMPTION OF BLAST IS 0.37" AND "THE FLOW RATE OF CONCENTRATE IS 0.62" AND “OXYGEN ENRICHMENT OF THE BLAST IS 0.25".

RULE 3: "IF COPPER CONTENT IN THE CHARGE IS THE MINIMUM" AND "SULPHUR CONTENT IN THE CHARGE IS THE MEDIUM" AND "SILICON OXIDE CONTENT IN THE CHARGE IS THE MINIMUM" AND "MAGNETITE CONTENT 
IN THE CHARGE IS THE MINIMUM" THEN "THE CONSUMPTION OF BLAST IS 0.37" AND "THE FLOW RATE OF CONCENTRATE IS 0.62" AND "OXYGEN ENRICHMENT OF THE BLAST IS 0.31".

The production rules for all 89 experiments are made in the same way.

After Matlab performs all the necessary procedures in accordance with the selected fuzzy output algorithm (for example, the Mamdani algorithm), a fuzzy model of optimal control of the copper smelting process is presented in the graphical interface for viewing the rules (see figure 1). Thus, the interface shown in figure 1 is a model (algorithm) of optimal control, which can be used to simulate various modes for all possible combinations of input variables.

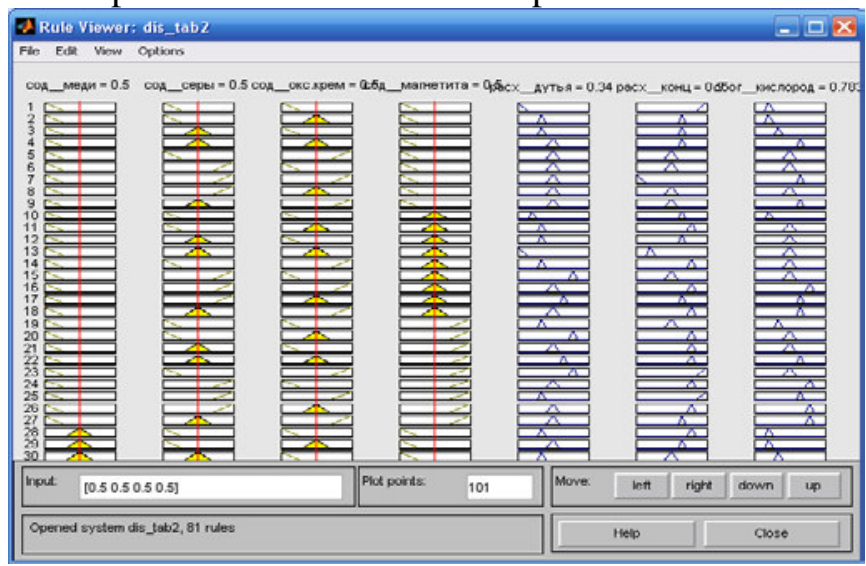

Fig. 1.Fuzzy control model (algorithm)

\subsection{Synthesis of neural network model}

Instead of fuzzy models, neural networks can also be used to model the process of managing modes in the PV. For training the neural network, the results of 89 experiments from the PFE planning matrix were entered into the Matlab program, an example of such input is shown in figure 2. Output variables (control actions) were entered using the "Datatarget" window (figure 3). 


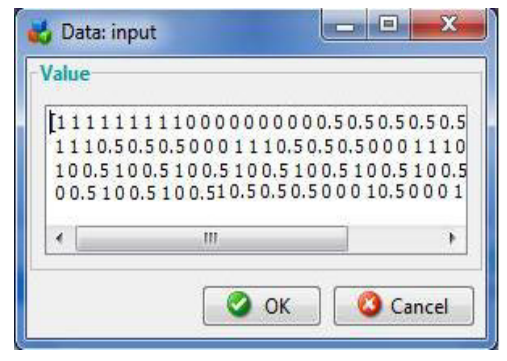

Fig. 2. Creating input data

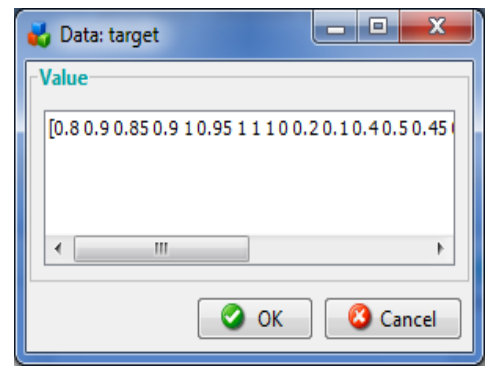

Fig. 3. Creating target data

At the next step a neural network was created. The input data field specified pre-created data, set the type of neural network, and selected a perceptron (Feed-Forward Back Propa-gation) with 10 sigmoid (TANSIG) neurons of the hidden layer and one linear (PURELIN) neuron of the output layer. The training was performed using the Levenberg-Marquardt algorithm, which implements the TRAINLM function. The error function is MSE, and the number of layers is 2 , respectively. The progress and results of the training are shown in figures 4 and 5.

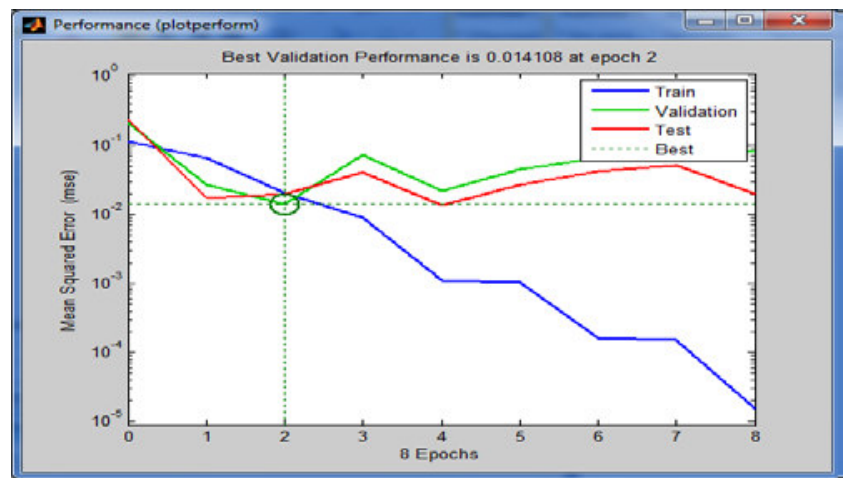

Fig. 4. Neural network learning progress 


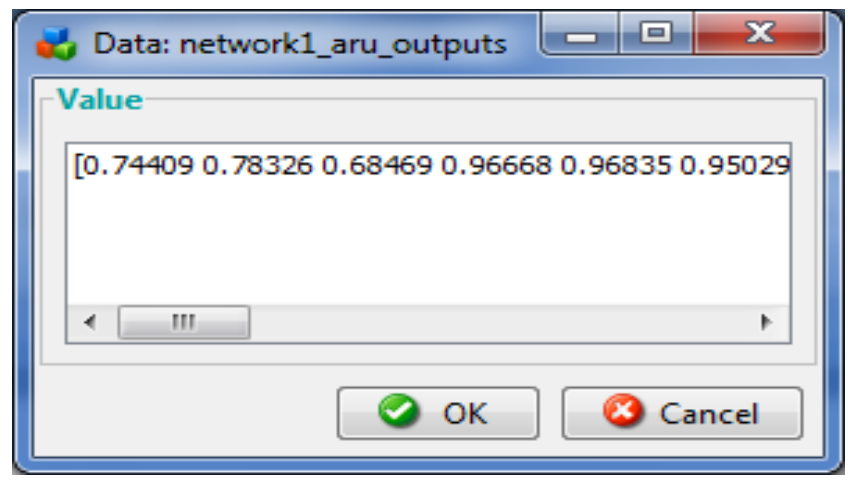

Fig. 5. The result (output) of training a neural network

\subsection{Synthesis of neuro-fuzzy control model}

Another method of modeling using intelligent technologies is hybrid models, such as neuro-fuzzy networks, which should combine all the advantages of the two methods listed above.

These researches can be performed using MATLAB. To do this, MATLAB has an ANFIS editor that allows you to create or load a specific model of an adaptive neuro-fuzzy inference system, train it, visualize its structure, change and configure its parameters, and use the configured network to get fuzzy inference results

The network presented in figure 6 is a model for managing the process of smelting in a liquid bath (SLB) using neuro-fuzzy algorithms.

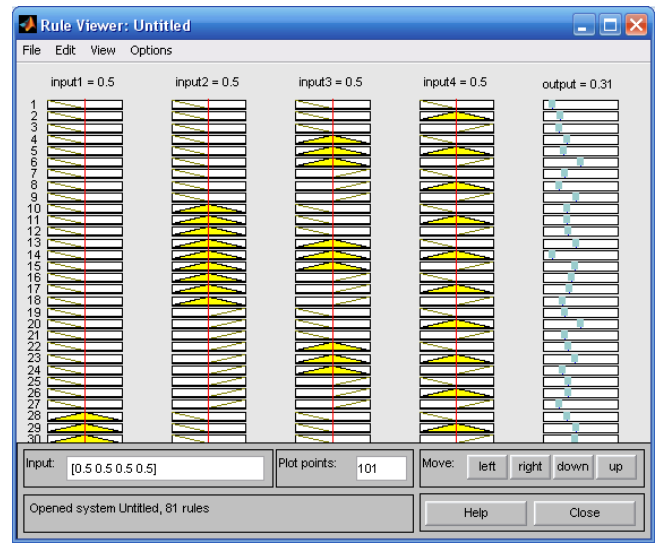

Fig. 6. Neuro-fuzzy model 
In the future, this model can be used to calculate output variables for any changes in input variables.

\subsection{Results of research of intelligent models}

The obtained control models allowed us to simulate various modes of process management depending on the composition of the run-ofmine charge.

The simulation results are listed in table 4 .

Table 4

Comparative estimation of absolute error for different methods

\begin{tabular}{l|c|c|l}
\hline \multirow{2}{*}{ Modelling method } & \multicolumn{3}{|c}{ Absolute error \% } \\
\cline { 2 - 4 } & $Y_{1}$ & $Y$ & $Y_{3}$ \\
\hline 1. Design of experiment & 15,53 & 33,01 & 12,68 \\
\hline 2. Fuzzy algorithms & 4,462 & 4,997 & 3,612 \\
\hline 3. Neural network algorithms & 2,26 & 2,8 & 2,78 \\
\hline 4. Neuro fuzzy networks & 1,333 & 1,002 & 1,241 \\
\hline
\end{tabular}

The table 4 shows that the experiment planning method cannot be used due to unacceptably high values of absolute errors: from $12 \%$ to $33 \%$. Intelligent models showed their advantage: from $1.0 \%$ to $5.0 \%$, while the best method is neuro-fuzzy networks (from $1.0 \%$ to $1.3 \%$ ).

Finally, the conducted researches have shown high efficiency of the control algorithms, obtained by using the artificial intelligence methods. In comparison with classical methods of building analytical and statistical models, methods based on the knowledge and experience of human experts allow creating optimal control systems for complex technological processes significantly easier, faster and more efficient.

3 Development and research of algorithms for diagnostics of equipment technical condition

Another important resource-saving tool is the ability to quickly assess the technical condition of equipment, which will allow timely preventing the emergency state of the main and auxiliary technological equipment. Let's consider an example of using AI methods to assess the technical condition of turbine units (TU).

The energy sector is currently characterized by an intensification of the use of capacities and resources of installed equipment. This can be achieved based on intelligent diagnostics of the operational state and modes of equipment use. The increasing responsibility of deci- 
sions made on the time of equipment commissioning has tightened the requirements for the quality of identification models, which are based on the information obtained during diagnostics of the TU condition. Their implementation in the conditions of the old forms of maintenance under the PPR system (planned preventive repairs) has become ineffective. There has emerged a problem of insufficient adequacy of diagnostic models and decision-making models for putting TU into repair or reducing the load, due to not using fuzzy information about the condition of equipment, as well as increasing the total uncertainty accumulated during operation.

In order to implement the above three-stage procedure for creating a diagnostic subsystem for the case of turbine units, a survey of operators-technologists and ITR of the turbine shop has been conducted, which showed that the main variables that characterize the state of the turbine unit as a whole are the following:

$X 1$ - thrust bearing vibration;

$X_{2}$ - plummer bearing vibration;

$X_{3}$ - thrust bearing babbit temperature;

$X_{4}$ - plummer bearing babbit temperature;

$X_{5}$ - the axial shift in the direction of the generator;

$X_{6}$ - axial shift in the direction of the "chair";

$X 7$ - relative expansion of the high-pressure rotor;

$X_{8}$ - relative expansion of the low-pressure rotor;

$X_{9}$ - pressure of hydrogen in the generator housing;

$X_{10}$-temperature of hydrogen in the generator housing;

$X_{11^{-}}$oil temperature after the oil cooler;

$X_{12}$-pressure in the discharge chamber of the high-pressure cylinder;

$X_{13}$ - hot steam temperature; X14- hot steam pressure;

$X_{15}$-vacuum drop in the condenser;

$X_{16}$-metal temperature in a high-pressure cylinder;

$X_{17}$-metal temperature in the low-pressure cylinder.

All these variables are controlled by devices, which makes it possible to synthesize an automated system for rapid diagnostics of the technical condition of turbo units.

In accordance with the methodology proposed in this paper, a matrix for planning a complete factor experiment (PFE) is compiled for the synthesis of an intelligent model of diagnostics and forecasting. 
However, in this case, it will be necessary to conduct a huge number of "thought" experiments, for example, for a three-level assessment, the number of experimental points will be $\mathrm{N}=3^{17}$, which is completely unrealistic to implement.

Therefore, the decomposition of the problem of forming the PFE planning matrix is carried out. For this purpose, the influence of diagnostic features $X_{1}-X_{17}$ on the state of the turbine unit as a whole, but on its individual main parts is evaluated, which will reduce the dimension of the PFE matrix. Taking into account that the steam turbine is a single-shaft two-cylinder unit designed for direct drive of the generator, it is proposed to consider the turbine unit as a set of the following main elements located on one shaft: a plummer bearing (PB); a high-pressure cylinder and rotor (HPC); a low-pressure rotor and cylinder (LPC); an alternating current generator (ACG); a thrust bearing (TB). In addition, due to the special danger, the including the hydrogen supply system (HSS) in the generator housing is planned. HSS will be considered as a separate element, not connected to the rest by a single shaft (figure 7).

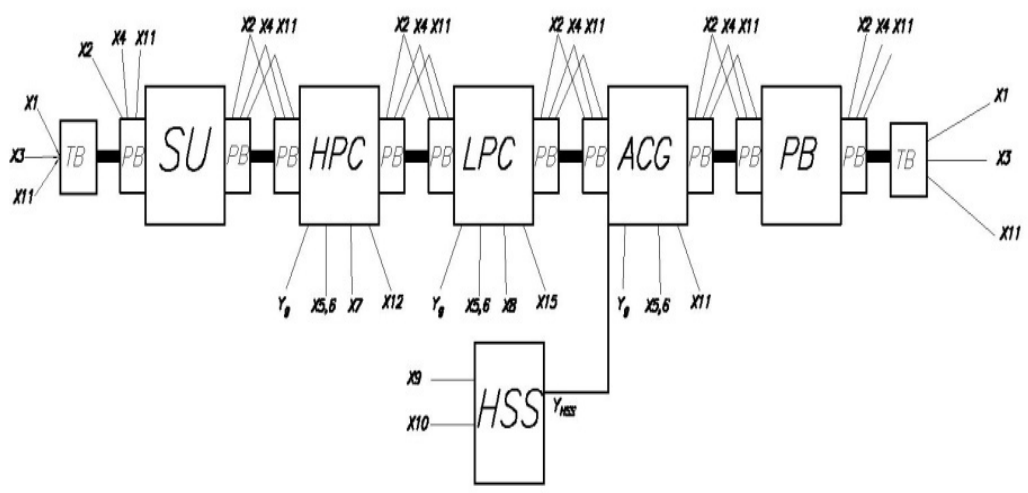

Fig. 7. Turbine unit elements and impact on technical condition of TU

Figure 7 shows that the technical condition of bearings is affected by vibration, babbit temperature and oil temperature, while the technical condition ratings of 10 bearings $\left(Y_{\mathrm{PB}}\right.$ и $\left.Y_{\mathrm{TB}}\right)$, in turn, are diagnostic features for assessing the technical condition of the HPC, LPC and ACG. Considering the fact that is these elements of the turbine 
are equally affected by the condition of both the plummer and thrust bearings. In this regard, it is proposed to take into account the assessment of the technical condition of only one of the 10 bearings that has the worst rating value (let's denote the value of this assessment in NP ).

As a result, taking into account the assessment of the technical condition of only one of the 10 bearings that has the worst rating value (this rating is indicated by $\mathrm{Y}_{\mathrm{g}}$ ), there is a reduction in the number of diagnostic features per unit for each of the three main elements: HPC, LPC and ACG.

The assessment of the technical condition of the most dangerous hydrogen supply system ( $\mathrm{Y}_{\mathrm{HSS}}$ ) depends on the values of the pressure and temperature of the hydrogen in the generator housing. At the same time, $Y_{\mathrm{HSS}}$ together with other diagnostic features $\left(X_{5,6}, Y_{\mathrm{g}}\right.$, and $X_{11}$ ) can serve as initial data for evaluating the technical condition of the generator as a whole.

Wherein, the axial shift to the generator $\left(X_{5}\right)$ and the axial shift to the chair (X6) are mutually exclusive factors, i.e. the shift can be carried out either in one direction or in the other, so these two factors were combined into one - $X_{5,6}$, which reduced the number of diagnostic features by one for each of the three main elements: the HPC, LPC and ACG.

In addition, the variables $X_{16}$ (metal temperature in the highpressure cylinder) and $X_{17}$ (metal temperature in the low-pressure cylinder) are used for evaluating the technical condition of the HPC and the LPC only in the process of preparing them for start-up during heating. In the course of normal operation of the turbine unit, they are not even controlled by a person, so they have been excluded from the number of diagnostic features, thereby further reducing the dimension of the problem that being solved.

The variables $X_{13}$ (hot steam temperature) and $X_{14}$ (hot steam pressure) are purely technological, depending on the physical state of the steam coming from the boiler shop. These variables cannot be used to assess the technical condition of a HPC or LPC, so they have been also excluded from the number of diagnostic features.

Thus, taking into account the reduction of diagnostic features, the assessment of each of the main elements of the turbine unit (TU) can be estimated only by four diagnostic features (figure 4.1), conse- 
quently, the number of "thought" experiments for each of the parts of the TU (HPC, LPC and ACG) will be $N=3^{4}=81$, which is quite simple to implement.

As a result of this research, four types of models for diagnosing the technical condition of the turbine unit have been synthesized. Comparative table 5 of these models illustrates that the best result was shown by neuro-fuzzy networks of $0.8 \%$ error, which is a very good result compared to traditional methods (the experiment planning method showed $6.7 \%$ error).

Table 5

Final comparison table for different methods

\begin{tabular}{l|l}
\hline \multirow{2}{*}{ Modelling method } & Absolute error \% \\
\cline { 2 - 2 } & $Y$ \\
\hline 1. Fuzzy algorithms & $1,15101 \%$ \\
\hline 2. Neural network algorithms & $1,146136 \%$ \\
\hline 3. Neuro fuzzy networks & $0,88102 \%$ \\
\hline 4. Design of experiment & $6,75 \%$ \\
\hline
\end{tabular}

Due to the best result of the neuro fuzzy model, all further research will be conducted only on the basis of this model.

\subsection{Sensitivity analysis of a neuro-fuzzy model}

Sensitivity means that a small change in the input parameters leads to a change in the system's property index that can be detected under conditions of measurement error. The purpose of sensitivity analysis is to compare the impact of various factors on the result of solving the modeling problem.

The integrated circuit sensitivity analysis:

- selection of factors, a slight change in which can have a significant impact on the result from the point of view of the researcher;

- setting the nominal and maximum (lower and upper) values of the selected factors;

- solving the modeling problem in different ranges of source data for all selected limit values of factors;

- building dependencies for the sensitivity of the problem solution for all factors and ranges of changes in the source data.

Figure 8 shows the results of sensitivity estimation when all 4 input variables are changed by a value of 0.02 . 


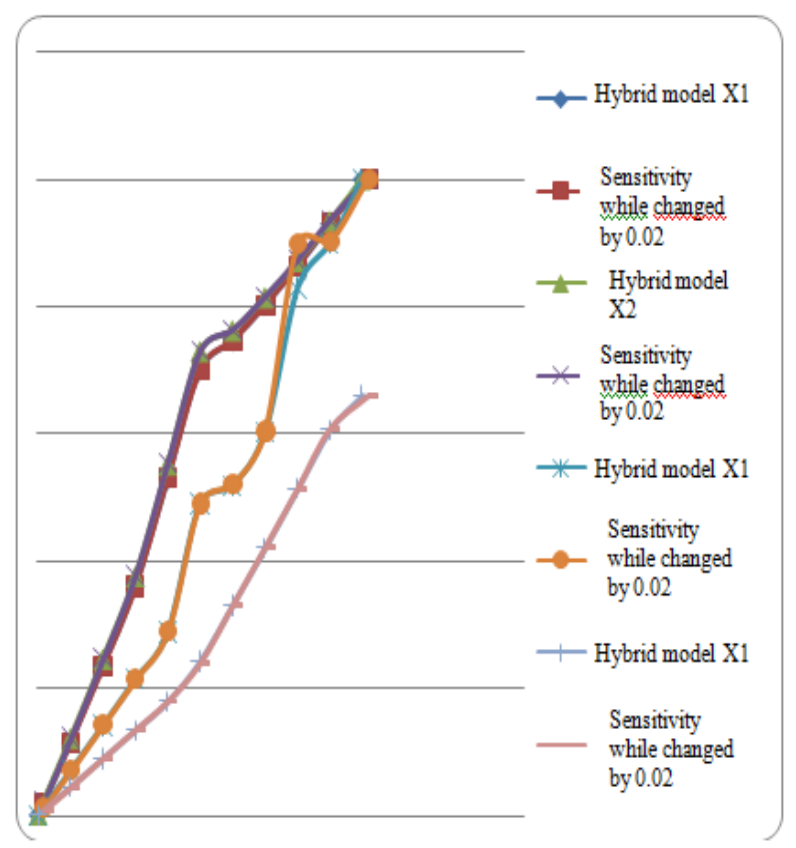

Fig. 8. Estimation of the sensitivity of the neuro-fuzzy model

A slight 0.02 change of input variables affected the output variables as shown in Figure 7. When diagnosing the technical condition of HPC change the values of input variables at 0.02 according to the logic and physics of the technological process should not greatly poulet on the technical assessment of HPC because of their insignificance. Fig. 2 shows that the curves before and after changes in the input variables by 0.02 slightly differ from each other, which fully explains and proves the high stability of the neural network model for diagnosing the technical condition of the HPC. In this case, thebI neural fuzzy model is most sensitive to changes in the value of the relative expansion of the high-pressure rotor $X_{3}$ and $X_{2}$ of the axial shift towards the generator or the front chair.

3.2 Analysis of the results of modeling the assessment of the condition of the high pressure cylinder

It is proposed to consider simulations of estimating the state of the HPC $(Y)$ for different rates of input variables: $X_{1}, X_{2}, X_{3}$ and $X_{4}$ (figure 9). 


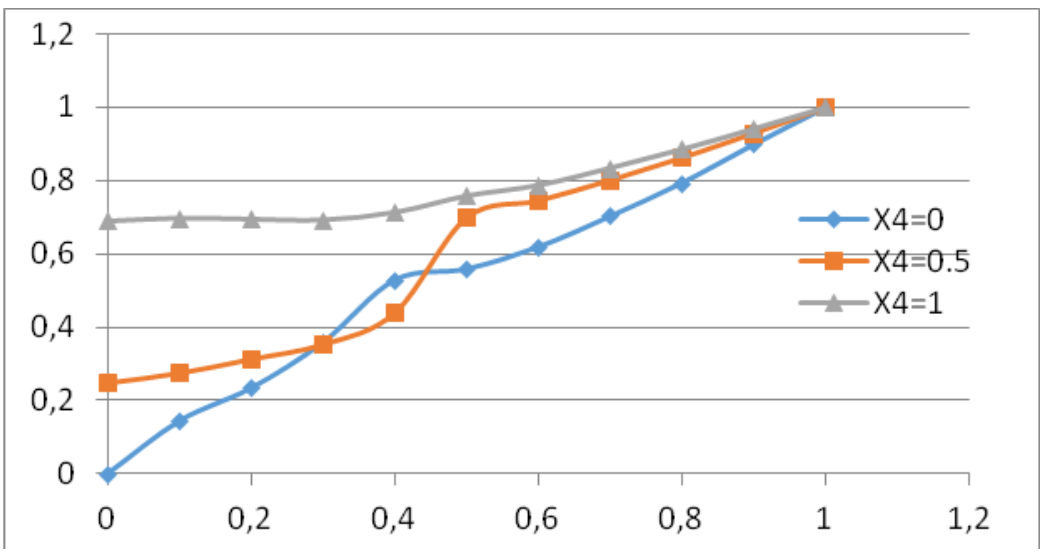

Fig. 9. Simulation Results for evaluating the technical condition of the High pressure cylinder depending on changes in the pressure of the discharge chamber at $X_{2}=0$ and $X_{3}=0.5$

Figure 9 shows the simulation results for evaluation technical condition of HPC (Y) depending on changes of the general condition of the thrust and plummer bearings $\left(X_{1}\right)$ for various rates of the discharge pressure chamber HPC $\left(X_{4}\right)$, equal to the maximum $\left(X_{4}=1,0\right)$, medium $\left(X_{4}=0.5\right)$ and minimum $\left(X_{4}=0\right)$ rates. The simulation was performed at nominal rates of the axial shift towards the generator or «front chair» $\left(X_{2}=0\right)$ relative expansion of the high-pressure rotor $\left(X_{3}=0\right)$.

The figure 9 illustrates, the maximum value $(Y=1)$ of the technical condition characteristic of the HPC is at the maximum rates of the general condition of the thrust bearing $\left(X_{1}=1\right)$ at any pressure rates in the discharge chamber of the HPC $\left(X_{4}=0,0.5,1.0\right)$. This is quite understandable - since the general condition of the thrust bearing equal to $\left(X_{1}=1\right)$ is unacceptable and inevitably leads to a stop of the turbine unit, according to the operating instructions [1]. When the normal pressure in the discharge chamber of the HPC $\left(X_{4}=0\right)$ and when the normal state of the thrust bearing $\left(X_{1}=0.0\right)$ the overall technical condition of the HPC is the best $(Y=0.0)$, and if the general technical condition of the thrust bearing deteriorates to $\left(X_{1}=0.5\right)$, the overall technical condition of the HPC is satisfactory $(Y=0.559)$

From figure 9 it is also seen that at moderate pressure in the discharge chamber $\left(X_{4}=0.5\right)$ is characterizing the wear of the discharge chamber HPC, and the best rates of the general technical condition of bearing 
$\left(X_{1}=0\right)$ general assessment of the technical condition of HPC satisfactory $(Y=0.249)$ and increasing, that is, deterioration of the general technical condition of thrust bearing $X_{1}$, the overall assessment of the technical condition of HPC is deteriorating, and is at $\left(X_{1}=0.5\right)$ is the overall technical assessment of HPC is equal to $(Y=0,7)$, this means that the unit is no longer in its normal technical condition and requires increased attention from the operator who controls the turbine unit, and if necessary, a detailed inspection of the unit without stopping it.

Further analysis of figure 8 shows that at maximum pressure in the discharge chamber $\left(X_{4}=1\right)$, and at nominal rates evaluation of the technical condition of the thrust bearing $\left(X_{1}=0.5\right)$, the overall technical condition of HPC is distinctly out-of-band mode $(Y=0,69)$, which is characterized by the poor condition of the discharge chamber of HPC, it is necessary to reduce the pressure in the discharge chamber HPC, by offloading that is, the reduction supplied to the steam turbine in order to prevent further destruction of the discharge chamber. And when the overall assessment of the technical condition of the thrust bearing is equal to $\left(X_{1}=0.5\right)$, the overall technical condition of the HPC is equal to $(Y=0.759)$, which is unacceptable for long operation of the turbine unit in these conditions.

The foregoing discussion has shown that the pressure value, that is, the technical condition of the high-pressure chamber of the HPC $\left(X_{4}\right)$ is not critical, since it is always possible to unload the turbine and thereby reduce this indicator. In this case, the value of the indicator of the general technical condition of the thrust bearing is critical for the overall condition of the turbine unit, because it is impossible to reduce this indicator when the turbine is running.

Figure 10 demonstrates the modeling results of assessment of the technical condition of the HPC $(Y)$, depending on the change in the rates of the axial shift in the line of the generator or front chair $\left(X_{2}\right)$, for different rates of the pressure of the discharge chamber of the HPC $\left(X_{4}\right)$, equal to the maximum $\left(X_{4}=1.0\right)$, average $\left(X_{4}=0.5\right)$ and the minimum $\left(X_{4}=0.0\right)$ value with nominal rates of the relative expansion of the high-pressure rotor $\left(X_{3}=0.0\right)$ and the general condition of the plummer bearing $\left(X_{1}\right)$ 


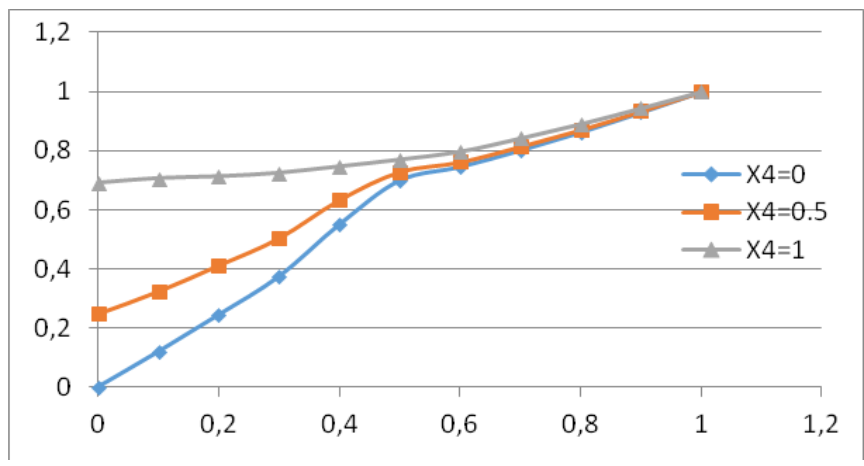

Fig. 10. Simulation results for evaluating the technical condition of the HPC $(Y)$ depending on changes in the rates of the axial shift towards the generator or front chair $\left(X_{2}\right)$ and at $X_{1}=0$ and $X_{3}=0$

Figure 10 shows that at minimal rates of discharge pressure chamber HPC $\left(X_{4}=0.0\right)$ technical assessment of HPC is deteriorating with increasing axial shift in the direction of the generator or of the front seat $\left(X_{2}\right)$. When $\left(X_{2}=0.5, X_{4}=0.0\right) Y=0.7$ a at $\left(X_{2}=1.0\right) Y=1.0$. Almost the same thing can be seen on the other two curves for estimating the technical condition of the HPC, which have different initial data depending on the pressure rates in the discharge chamber of the HPC. When $\left(X_{4}=0.5, X_{2}=0.0\right) Y=0.249$, when $\left(X_{2}=0.5\right) Y=0.729$ at $\left(X_{2}=1.0\right)$ $Y=1.0$ and finally at $\left(X_{4}=1.0, X_{2}=0.0\right) \quad Y=0.69$, at $\left(X_{2}=0.5\right) \quad Y=0.769$ and at $(\mathrm{x} 2=1.0) Y=1.0$.

The analysis of figure 10 allows concluding that the value of the axial shift towards the generator or the front chair $\left(X_{2}\right)$ is more critical than the pressure in the discharge chamber $X_{4}$. Since according to the operating instructions of the turbine unit - when the value of the axial shift in the line of the generator or the front chair increases and it is impossible to normalize it in the shortest possible time, the emergency shutdown of the turbine unit occurs.

During normal operation of the turbine unit, the axial shift is a fairly stable value, but with sharp changes in the parameters of the sharp steam, this indicator goes beyond the set value.

This is illustrated in figure 11 


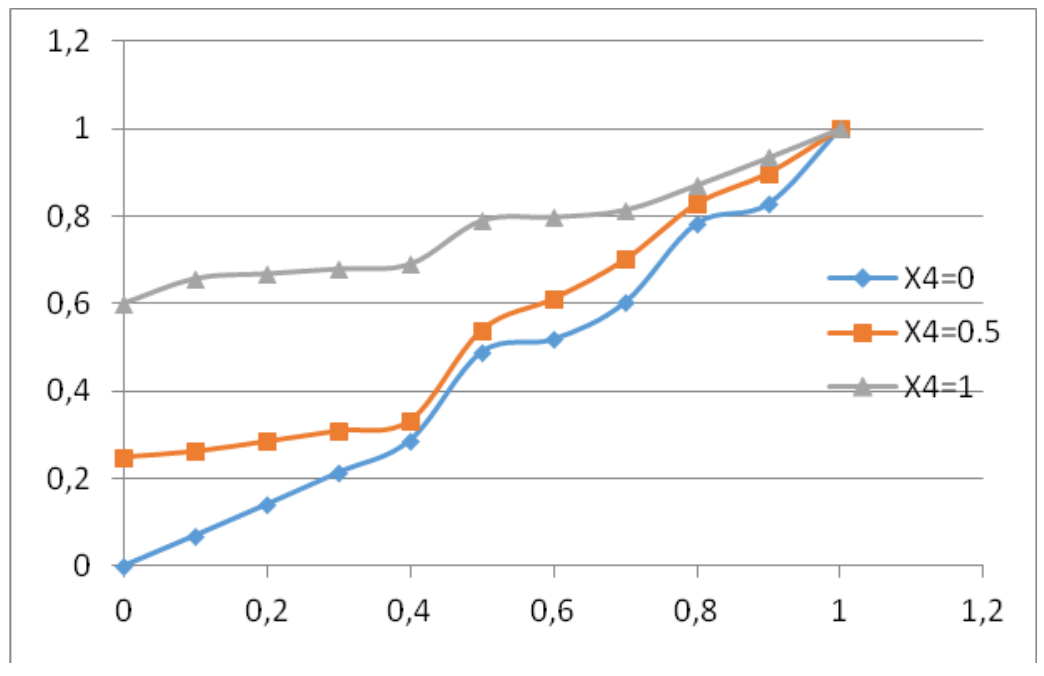

Fig. 11. Simulation results for evaluating the technical condition of the HPC $(Y)$ depending on changes in the rates of the relative expansion of the high-pressure rotor $X_{3}$ and with nominal rates $X_{1}=0$ and $X_{2}=0$

As shown figure 11, which suggest how the overall assessment of the technical condition of the HPC $(Y)$ changes, depending on the change in the relative expansion of the high-pressure rotor $\left(X_{3}\right.$ from 0 to 1$)$ and at the nominal rates of the general state of the thrust and plummer bearing $\left(X_{1}=0\right)$, the axial shift in the line of the generator or the front chair $\left(X_{2}=0\right)$ and at the rates of the pressure in the discharge chamber equal to $X_{4}=0, X_{4}=0.5$ and $X_{4}=1$.

Figure 11 shows that for different pressure values in the highpressure discharge chamber, $X_{4}=0.0, X_{4}=0.5$ and $X_{4}=1$ of the initial points of the curves correspond to the value of the general technical status of HPC $Y=0, Y=0.249$ and $Y=0,6$. While increasing in the value of the relative expansion of the rotor high pressure $X_{3}$, and the values of the pressure in the high pressure chamber $\left(X_{4}=0.0,0.5\right.$ and 1$)$, the value of the general technical status of HPC $(Y)$ tend to 1 then to the emergency state at $X_{4}=0\left(X_{3}=0.5, Y=0.49\right),\left(X_{3}=1 \quad Y=1\right)$ with $X_{4}=0,5$ $\left(X_{3}=0.5, Y=0.54\right),\left(X_{3}=1, Y=1\right)$ with $X_{4}=1 \quad\left(X_{3}=0.5\right.$, and $\left.Y=0,79\right)$, $\left(X_{3}=1, Y=1\right)$. 
These data illustrate that the value of the relative expansion of the high-pressure rotor- $X_{3}$ is also critical when evaluating the overall technical condition of both the HPC and the entire turbine as a whole.

That meets the requirements of the operating instructions of the turbine unit. The expansion of the high-pressure rotor is accompanied by the same threats as the axial shift towards the generator or the front chair.

Thus, the simulation results of evaluation of the technical condition of HPC are reasonable in terms of physics of technological process unit and fully reflect the assessment of the experts - the operators of the turbines.

\subsection{Recommendations for liquidation of emergency situation HPC}

Further adopted the following graduation estimates the degree of closeness of the current state of HPC to the emergency situation:

$a$ - if the value of $Y$ ranges from 0 to 0.25 - HPC is in the normal running order;

$b$ - if the value of $Y$ ranges from 0.26 to 0.5 - emergency situation of HPC is possible, but more careful monitoring is required, and the necessary preventive actions of the operational personnel are given above;

$c$ - if the value of $Y$ ranges from 0.51 to 0.79 - it is a preemergency situation;

$d$ - if the value of $Y$ ranges from 0.8 to 0.1 , an emergency situation has occurred

Depending on the value of the assessment of the technical condition of the HPC, the operational diagnostics subsystem can make one of the following decisions:

- in case $(a)$ - do nothing;

- in case $(b)$ is to analyze possible causes of the deteriorating condition of HPC: to check the temperature of bearings and the temperature of the cooling water; check circulation lubrication and the operation of the oil pump; check the pressure in the discharge chamber HPC and in front of it; check to see that lines of heating of flanges and studs; check the position of the turbine shaft on special marks; 
- in case $(c)$ - depending on the results of analysis to produce one of the following: isolate the cause of the temperature rise of the bearings, and eliminate it according to instructions and the above measures; if necessary, reduce the steam flow to the turbine head to the normalization of the pressure in the discharge chamber HPC; if necessary, adjust lines of the heating flanges and studs;

- in the case of $(d)$ - act on the order of the shop technologist, shift Manager or Deputy head of the shop for operation or the person replacing him.

Similarly, it is possible to create a PFE planning matrix for evaluating the technical condition of other elements of the turbine unit: the Central heating unit, the PS, and the generator. Faults of this class are detected by the appropriate sensors, and the response to them is specified in the process instructions and can be duplicated on the monitor screen using the rapid diagnostics subsystem.

Thus, the proposed method of evaluating the technical condition of the turbine unit allows predicting the occurrence of emergencies at an early stage.

\section{Conclusion}

This paper describes the concept of creating intelligent control systems for technological processes. It presents the results of the development of intelligent algorithms for determining the key variables of the copper melting process in a liquid bath and algorithms for evaluating the technical condition of turbine units.

Experimental algorithms were created using fuzzy logic, neural and hybrid networks, which enable us to formalize the knowledge of experienced technologists.

The check of the adequacy of the algorithms showed that the minimum discrepancy between expert data and model data is inherent in the neuro fuzzy networks algorithm.

The absolute error value for the test sample of expert and model data of both applications (the copper melting process in a liquid bath and for evaluating the technical condition of turbine units) was $1,3 \%$ and $0.88 \%$, respectively. 
In its turn, this indicator for the classical method of design experiment made $15 \%$ and $6.5 \%$. This proves the advantage of intelligent technologies over classical methods in identifying mathematical models of control objects.

Therefore, resulting algorithm can be used as an autonomous expert system or integrated into the overall control system of the described technological processes. This eliminates the influence of the human factor and enable us achieving high technological indicators and predicting the occurrence of emergencies at an early stage.

The arguments that have been presented in this paper suggest that the using of intelligent systems in the control of mining and processing of minerals and in the diagnostics of equipment allows building high-quality control systems reducing the resource consumption of production.

References

1. Timothy, J. Ross. (2017). Fuzzy Logic with Engineering Applications. USA: John Wiley and Sons.

2. Laurene, Fausett. (1994). Fundamentals of Neural Networks: Architectures, Algorithms And Applications 1st Edition. India: Pearson

3. Vanyukov, A.V., Bystrov, V. P., Vaskevich, A.D. and others.(1988). Melting in a liquid bath. Moscow, M: Metallurgy

4. Bozhko, V.V., Kovalenko, A.N., Lyapunov, V.M., Khomenok, L.A. (2016). Operational diagnostics of the thermal state and efficiency of steam turbines of TPP and NPP. DOI:10.1134/S0040363616030036

5. Suleimenov, B. A, Sugurova, L. A., Suleimenov, A. B. (2016). Intelligent systems of optimal control and operational diagnostics (methods of synthesis and application). Almaty: Shikula 2016.

6. Roger Jang, J. S. (1997). MATLAB fuzzy logic toolbox: User's guide.

7. Smirnov, D., Komashinskij, V. (2004). Neural networks and their application in the control and communication system. Moscow: Telekom.

8. Yu-JieWang. Ranking triangle and trapezoidal fuzzy numbers based on the relative preference relation. (2015). Applied Mathematical Modelling:39:586-599. https://doi.org/ 10.1016/j.apm.2014.06.011. 